\title{
Psychological Assessment Data Processing Model Based on Neural Network Theory
}

\author{
Chen Xiaowang
}

Guilin Medical University

*email: 24547350@qq.com

\begin{abstract}
Neural networks are real rough neural network simulation, it is fault tolerant, anti-interference, high efficiency and other characteristics, with learning, memory and other cognitive functions powerful and therefore suitable for simulation of psychology in many areas.

In this paper, the data from College Students Mental for the study, based on SCA (Service Component Architecture) technology to complete the establishment and management platform, based on theoretical knowledge of neural networks for processing the data, thus completing the psychological evaluation data models, different psychological data processing method evaluation provides a theoretical and technical research platform.
\end{abstract}

Keywords: Neural networks, psychological evaluation, data processing, Service Component Architecture

\section{Introduction}

The researcher neural network model is a physical device or a computer program, real rough neural network simulation. From the network architecture and storage processing methods neural network model, the neural network model is a parallel distributed processing system, which consists of many simple processing units linked together with each other according to a specific task, by changing the information processing unit coupled to the process weights to achieve the desired output of the network $^{[1]}$. A neural network model includes two aspects: (1) determine the network structure. Includes a number of network cell layer (the number of input units and output unit number) used, the connection between the cell layer (interconnection between layers, the inner layer units are connected, the input layer and the output unit cell layer loop connection, etc.) and the level of activation function unit (hard limit function, linear function, logarithmic function, multinomial logistic functions, etc.). (2) determine learning rule. Learning the rules is to modify neural network weights and bias value method (also referred to as training algorithm), it depends on the choice of model to solve the problem, the scale, performance requirements model. Common law has Hebb rule, $\triangle$ law and other laws and BP. In accordance with the training route network to achieve the goal of learning the law it can be divided into supervised learning, reinforcement learning and unsupervised learning categories ${ }^{[2]}$. Supervised learning is to give advance training methods network input and standard output value; reinforcement learning is to give network performance evaluation level training methods; unsupervised learning is a training method to automatically adjust the weights and bias values according to the network input.

\section{Psychological Evaluation System Design}

Psychological evaluation is an essential part of many colleges and universities enrolled students to understand the psychological, as well as carry out a necessary work to carry out the daily work of colleges and universities, with the increase in the number of college students into the school, the need for timely to make their mental health screening, face huge the amount of data, so the need to establish a web-based platform for open data evaluation system to improve the efficiency of data collection $^{[3]}$. While providing a more in-depth psychological analysis of data, providing sufficient data and decision support for colleges and universities to carry out psychological education and psychological counseling. With the development of computer information technology, in order to make better use of computer technology to combine Internet information psychological survey of the 
current problem is many colleges educators face. To solve this problem, not only need to understand the structure of mental health, we must also be present according to the characteristics of students, designed to meet the mental health test questions. Internet technology platform, to achieve the evaluation of software development and maintenance, as well as later data analysis, is now psychometric network. This is also the path of development of Psychological educators must go. Psychological evaluation systems described herein use of B / S and C / S mode and cross-architecture used, data acquisition and data analysis is divided into two modules functionality. Data acquisition module for students, by students through a network of independent psychological evaluation; data analysis module for the College Counseling Center management staff, responsible for gathering up psychological evaluation data for statistical analysis. Wherein the data analysis module in addition to the traditional functions contain information management system, the increase of data mining functions, primarily using data mining techniques for psychological evaluation data mining depth analysis to identify than the huge amounts of data implied by law and information.

\subsection{System Architecture}

The system uses B / S mode and C / S mode and cross with the architecture. Different functional modules for the system's own characteristics, security and interoperability requirements are relatively high, a large number of users, the use of location widespread psychological evaluation data acquisition module designed for B / S mode; will require high data security, data computationally intensive, interactive, small number of users, the use of a fixed place of psychological evaluation data analysis module designed for $\mathrm{C} / \mathrm{S}$ mode. Cross with two modes, full use of their advantages to construct a B / S mixed mode with the C / S.

\subsection{System Development Platform}

Before the development of the system used in the system and technology platform selection is very important, not only makes good platform for future expansion of the system becomes easy, and maintenance of the system can be facilitated, based on these considerations. Psychological evaluation model based on neural network theory with a relatively mature and stable technology and service-oriented architecture SOA Service Component Architecture SCA ${ }^{[4]}$.

SOA (Service-Oriented Architecture) is a component model, its components can be defined as a module to be defined as a system, SOA component abstracted as one of service, through a unified interface and the link between the service contract. Service interface is defined in a neutral manner, and it independent of implementation services, hardware platforms, operating systems and programming languages. This allows the system to build SOA, scalability and portability stronger, but also makes building systems in a variety of such services can be unified and universal way to interact reusability, module or system stronger reduce the cost of doing the conversion or write code. SCA (Service Components Architecture) is a SOA-based specification, which simplifies the implementation of business service component programming model, these components can use different programming languages for enterprise applications, SCA also provides critical some infrastructure, like security , transactions, reliable calls, etc., which for the purposes of developing enterprise applications become very convenient.

SCA to achieve development and application system is divided into two steps and assembly, after the application of the system code, business functions can be abstracted as the range of services provided. Thus, this can be a range of services according to business logic sequence assembled into solutions based on specific business needs.

SCA is a member on the basis of the work, it is the constituent units of SCA. To achieve this is to provide member business function code fragment. The abstract interface to business functions as services provided to other member calls. Meanwhile, the member in the implementation process if rely on the services provided by other members, these dependencies are called references. Member during implementation may use some of the data that can be set in the properties. 


\section{Neural Network Analysis}

Based on neural network module provides a runtime framework to support the release of SCA components, neural network interface module to implement SCA assembly model. By configuring the instance to achieve complete SCA component that provides the resulting destruction service, SCA of these services can be accessed directly by declaring an external service to complete. SCA references represent a service call another service. SCA integration is an implementation ${ }^{[5]}$. Type SCA component mainly refers to the implementation of SCA configurable interface is mainly used to define different business functions, these different business implementation is achieved through different services, primarily by reference to these services to the assembly, these services by implementing different interfaces to complete, SCA supports two types of interfaces: WSDL port Types and Java interfaces, usually usually use a Java interface. SCA component is the basic unit SCA domain, SCA domain by the service, group profiles, references, services, and connections between these constituent.

SCA component is the configuration of the instance, it can provide a service or services through calling by Reference Service. Service is a component to an external call themselves the service interface, Reference is to achieve its own service's dependence on external services, external services Wire references connected to their service, Property enables service explicitly set the data value to change configuration, the data value provided by the component can also be derived from container composite properties. As shown in Figure 1.

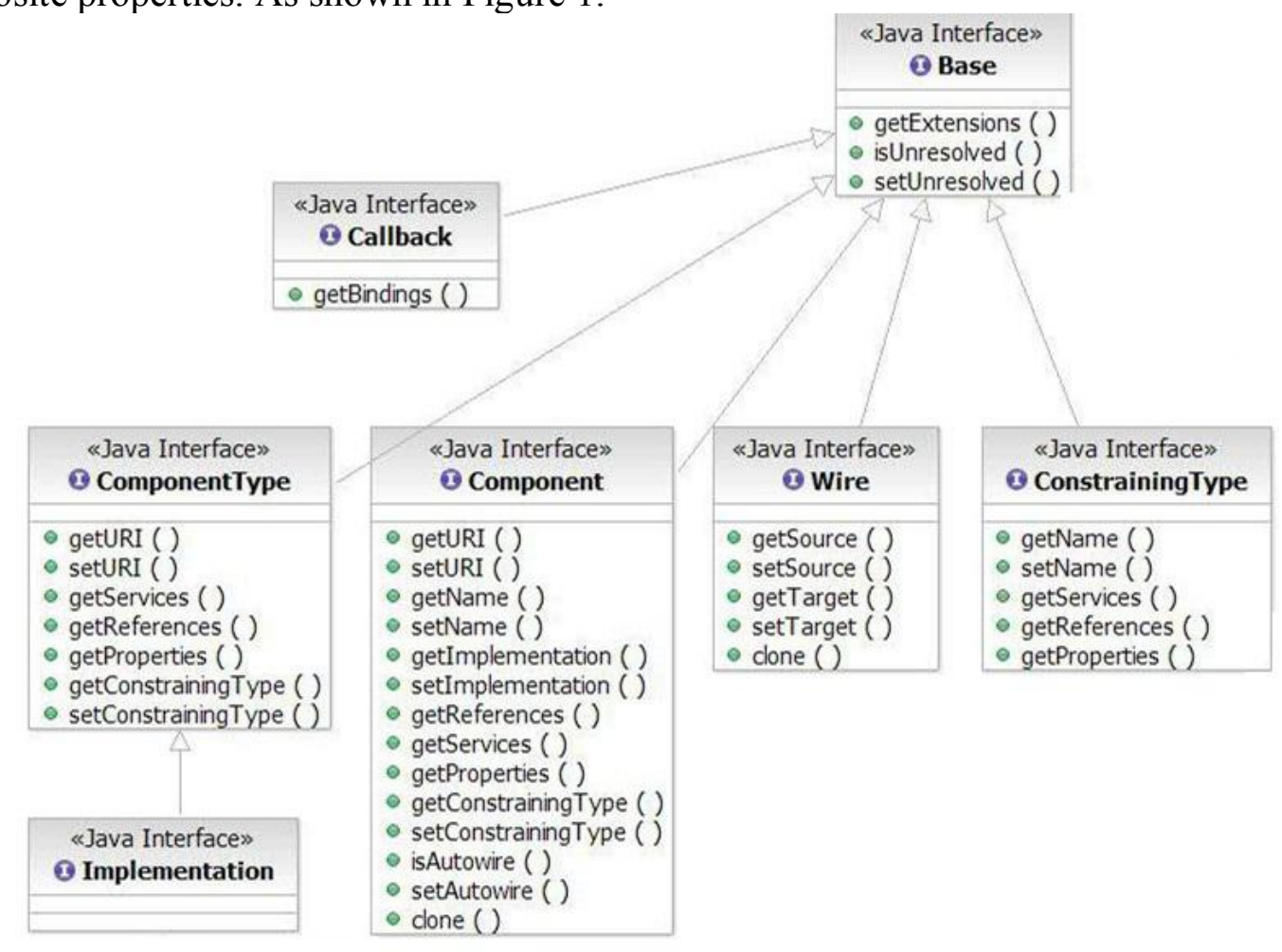

Figure 1. Java SCA Component Architecture Diagram Based on Neural Network

In the realization of the structure, when these services can be invoked through explicit@service way through java implementation. In some cases, does not necessarily have to use @service services through java achieved by implementing the type of services can also be inferred class itself. The next instance of a java implementation of java interfaces show services and the services provided through the interface.

Neural network modules SCA at run time services provided need to achieve the appropriate reference contract, and the contract service reference is made to develop the type attribute domain. Implementation instance attribute domain by setting the instance to complete. Scope determines when to inject operation. When the need to ask SCA instantiate the process at run time can provide a 
reference to the corresponding contract is wanted by the constructor parameter before adding @ reference to label, reference to the service contract specified by the constructor arguments. For not explicitly use@Reference or @Property annotation statement Java component implementation.

\section{Conclusion}

In this paper, the establishment of data processing by neural network platform, the platform services architecture choice using SCA services architecture, the platform through the creation and analysis, design evaluation system based on psychographic data neural network. Through code debugging, to prove the rationality of the system architecture and modular design. After the application of psychological evaluation model provides a theoretical analysis, but also reduce the workload of the psychological evaluation of personnel.

\section{References}

[1] Yang Jiangang.Artificial neural networks practical tutorial [M]. Zhejiang University Press, 2001.

[2] Piatetsky-Shapior, WJFrawley. Knowledge Discov-ery in Database [M]. Cambridge , Massachusetts, A:AAAI / MIT Press, 01: 158-165.

[3] Lei Xi-ling Ling Zhang Jing, Xie Tianbao. The prediction based on psychological problems genetic neural networks [J]. Computer Engineering, 2003, 29 (11): 132-133.

[4] Yin Qiang. Chongqing University Graduate Mental Health Survey Analysis and Countermeasures [D] Chongqing: Chongqing University, 2005.

[5] Xu Fangyuan. Based on bridge health of BP neural network evaluation, Chang'an University, 2006-11-06. 